\title{
COOL INFALLING GAS AND ITS INTERACTION WITH THE HOT ISM OF ELLIPTICAL GALAXIES
}

\author{
W.B. Sparks ${ }^{1}$ and F.D. Macchetto ${ }^{1,2}$ \\ ${ }^{1}$ Space Telescope Science Institute, 3700 San Martin Drive, Baltimore MD 21218*
}

\begin{abstract}
We describe work leading to the suggestion that interaction between infalling cool gas and ambient hot, coronal plasma in elliptical galaxies is responsible for emission filaments, and might remove the need for large mass depositions in cooling flows. A test of the hypothesis is undertaken - the run of surface brightness with radius for the emission lines - and the prediction agrees well with the data.
\end{abstract}

\section{INTRODUCTION}

Observations indicate that infall events are common in elliptical galaxies - high specific angular momentum gas discs, counter-rotating cores, normal dust properties, misaligned and warped dust lanes and Malin-Carter shells. This provides an obvious and likely origin for the extended low excitation emission nebulae and dust lanes often seen in active elliptical galaxies. For example, Sparks, Macchetto and Golombek (1989), Forbes et al. this conference, show in all four cases studied extended low excitation optical line emission associated with an infall or merger event.

There is however good evidence indicating that optical line emission is related to the $\mathrm{X}$-ray properties and in particular it preferentially occurs in those galaxies with short cooling time, $\mathrm{Hu}$ (1988). This has lead to the proposition that optical emission filaments arise in a cooling flow. Phillips et al. (1986) from spectroscopy of nuclei, and Trinchieri \& di Serego Alighieri (1989) from imaging found a similar correlation for normal elliptical galaxies, with higher $H \alpha$ line fluxes in galaxies showing $\mathrm{X}$-ray excesses.

This is a clear contradiction. On the one hand there is every reason to think that emission filaments arise from infall events, while on the other there is very good evidence that they are associated with the X-ray properties. Our observations of nearby radio ellipticals indicate that thermal conduction may provide the solution to these two apparently contradictory observational results by allowing the infall event to influence the $X$-ray properties. Cool infalling gas in the presence of effective thermal conduction can drain significant energy from a hot X-ray emitting balo thereby mimicing symptoms of the cooling flow. The energy transfer is adequate to power the optical line emission and infra-red radiation.

\section{PREVIOUS OBSERVATIONAL WORK}

Sparks et al. (1989) describe observations of NGC 4696, the dominant $\mathrm{CD}$ galaxy at the center of the Centaurus cluster. The emission-line filament system and dust lane in the central regions of this classical 'cooling-flow' galaxy are more plausibly explained by an external origin for the cooler material than by condensations in a cooling flow - the dust is spatially coincident with the ionized gas, its extinction variation with wavelength is normal, there is kinematic 
substructure in the galaxy, Danziger \& Focardi (1988), and there is a large low surface brightness linear feature which is reasonably explained as a tidal tail, Malin (1985).

Given that an external origin for the cooler gas is favoured, the question of how infalling gas would interact with the ambient hot medium arises, and particularly with regard to energy transfer from the hot plasma to the cool gas. Our observations of the dust lane place good constraints on the dust-lane covering factor which together with the published X-ray gas properties, Matilsky, Jones and Forman (1985), allow us to deduce an available energy input rate of $3 \times 10^{43} \mathrm{erg}^{-1}$ from saturated electron heat conduction. This compares well to a radiated flux of $1 \times 10^{43} \mathrm{erg} \mathrm{s}^{-1}$ in the far-infra-red, Jura et al. (1987), and to $0.9 \times 10^{43} \mathrm{erg} \mathrm{s}^{-1}$ from line emission, Sparks et al. (1989). Energetically, in other words, heat conduction could provide ample power for all the line emission and infra-red radiation we observe. However, if this occcurs, it represents a severe drain of energy from the hot gas - greater than the X-ray losses over a much larger region.

\section{COOL PROPOSITION}

Given the fundamental nature of an energy transport process such as electron conduction, i.e. if electron conduction is an effective transport mechanism in one hot plasma it is likely to be so in essentially all, we propose that the general explanation for low excitation optical filaments in $\mathrm{X}$-ray luminous elliptical galaxies is infall. The short X-ray 'cooling-times' then arise simply as a local depression of temperature caused by heat lost to the dust-lane/filament system.

Infall events are transient, and we propose that in the absence of infalling gas when the cloud has evaporated, formed into stars or been lost to the nucleus, that the hot gas will return to a stable configuration with the rather modest losses due to $\mathrm{X}$-ray emission being balanced by an ample heat supply from elsewhere in the cluster. D'Ercole et al. (1989) show that using nominal supernova rates alone there is greater energy input per unit time at the present than there is energy lost from $\mathrm{X}$-ray emission in all elliptical galaxies, and since our original proposition was that heat conduction is effective, then such energy input is available to the hot medium as a whole. Kochhar (1989) in fact shows enhanced supernova rates in active ellipticals, which may arise from star formation associated with the infall event and can assist the hot gas in returning to its equilibrium configuration. The criticism that for heat conduction models to be viable requires 'fine tuning' is not valid since we are considering a dynamic transient state in which the cool phase is forced only temporarily on the hot, stable long lived phase.

The heated nature of the debate and July weather in Baltimore lead us to suggest 'coolers' as a concise description of the process advocated.

\section{OBSERVATIONAL TESTS}

Having arrived at a very different interpretation of existing observations, we clearly need to investigate predictions and possible tests. For the present work, one particular test is described, namely the run of emission-line surface brightness with radius.

If the conduction model is correct, then the $H \alpha$ line flux should be directly related to the $\mathrm{X}$-ray halo properties, and without any recourse whatsoever to the optical data, it is possible to make a simple prediction of the emission-line surface brightness as a function of radius given the halo properties. Consistent surface brightness distributions have proven a severe problem 
area for the cooling-flow model, Heckman et al. (1989).

Two galaxies have been investigated. M87 is probably the best studied from the Xray point of view although the emission filament system is somewhat fragmentary. In the innermost regions though, it covers a wider area of the galaxy and the test may be carried out. Heckman et al. (1988 preprint) present a surface brightness profile for $H \alpha$ line emission which we calibrated using the information in that paper and the fluxes of Ford \& Butcher (1979). Schreier et al. (1982) give a simple description of the X-ray gas temperature and density. Using this and setting predicted surface brightness equal to $1 \%$ of the available saturated heat flux, $Q_{\text {sat }} \propto n_{\mathrm{e}} T^{3 / 2}$, we obtain the line shown in Figure 1. Outside of regions where seeing and the non-thermal nucleus are likely to confuse, the agreement is extremely good. There is no a priori reason why the two curves need be even remotely similar since the prediction was made entirely on the basis of the $\mathrm{X}$-ray atmosphere, yet they lie within a factor of two over two orders of magnitude in surface brightness.

The second galaxy investigated is NGC 1052 the well-know active southern radio elliptical. Here the $\mathrm{X}$-ray data are not so good, but the optical data are more extensive, Sparks et al. 1990 in preparation, Forbes et al. this conference. Forbes et al. summarise the good reasons for believing the gas in NGC 1052 is external in origin. In order to estimate a 'predicted surface brightness', we assumed a galaxy mass distribution given by the light, and solved for an isothermal hot atmosphere. There are more free parameters in this model: the temperature translating into a scale length and density related to the total $X$-ray flux, and an overall normalization corresponding to the efficiency of energy transfer. We can get a good description of the surface brightness profile for values of these parameters which are all reasonable, again at least to within factors of two or so $-T \approx 5 \times 10^{6} \mathrm{~K}$, central density $n_{0} \approx 5 \mathrm{~cm}^{-3}$ but falling quite rapidly, see Figure 2. In this case, other possibilities are also viable and in particular photoionization by the active nucleus is a strong contender.

We conclude, nevertheless, that even though this analysis does not prove the validity of the conduction hypothesis, the results are consistent with the model and that from the point of view of the test as initially set, the theory passes, and passes well.

\section{CONCLUSIONS}

- Observations suggest cool gas comes from infall.

- Observations, statistics and theory imply effective conduction would be energetically important for all of the $X$-ray gas, emission-line radiation and infra-red flux.

- If conduction does operate, infall events will profoundly affect the hot gas and the cool gas, and may dominate the physical state adopted by both phases. 
Figure $1 \mathrm{H \alpha}$ emission line surface brightness profile of M87 compared to $1 \% \times Q_{\text {sat }}$, the straight line. $\log r=2$ is $\approx 1.4$ arcsec.

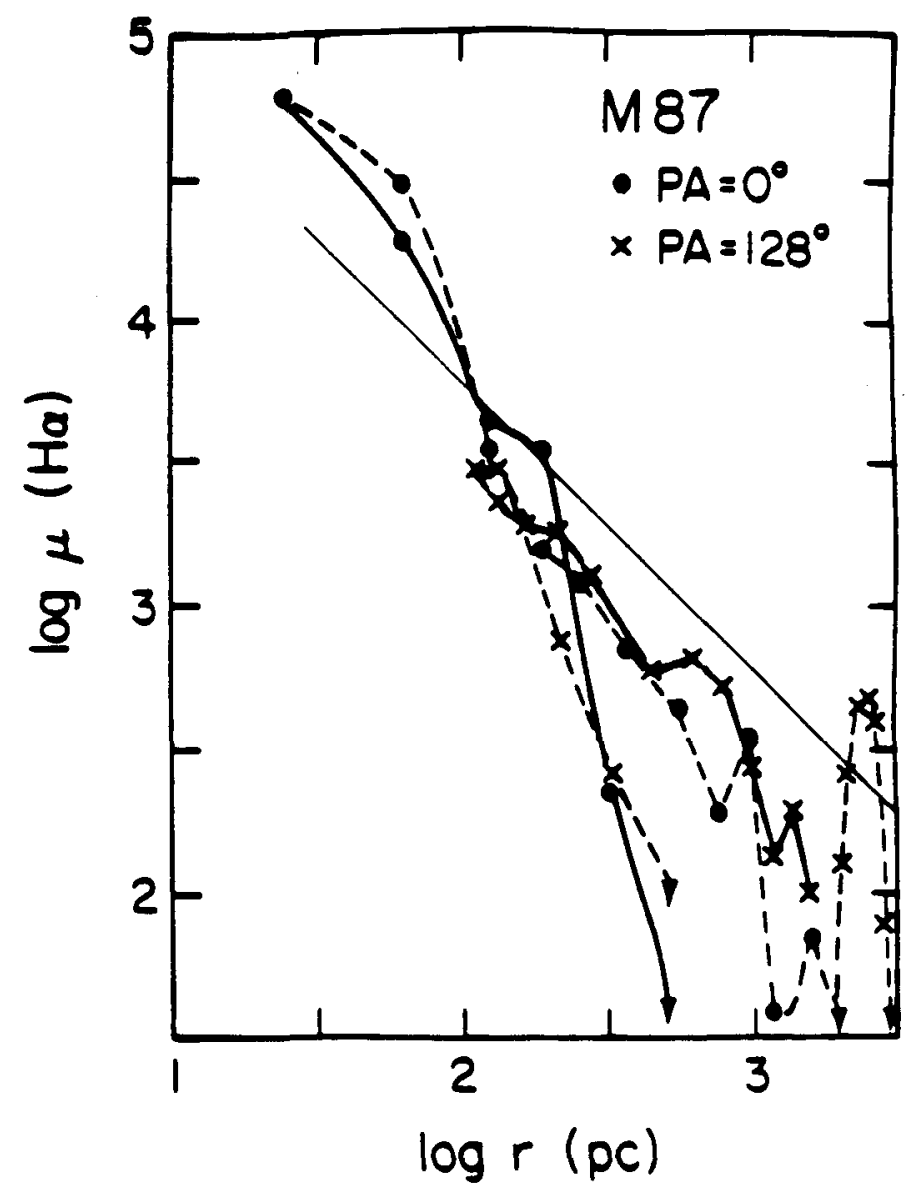

Figure $2 \mathrm{H \alpha}+[N I I]$ emission line surface brightness profile for NGC 1052 compared to a thermal conduction heating model. The upper and lower curves are estimates of the uncertainty and the data are cut off at $\approx \mathbf{4 0}$ arcsec.

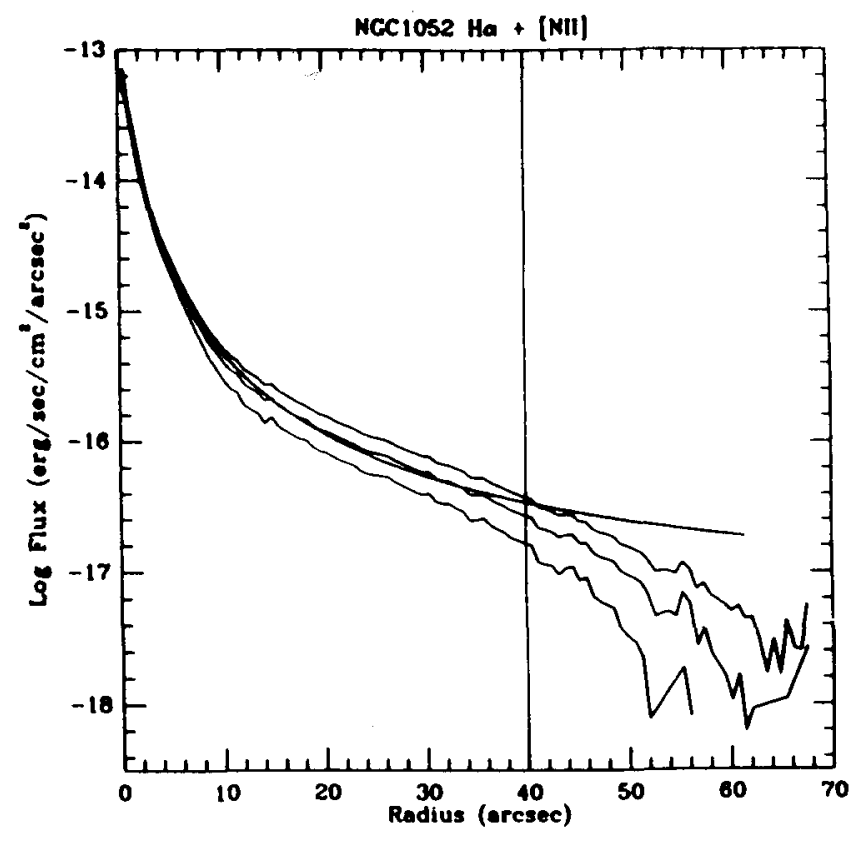




\section{References}

Danziger, J., Focardi, P. 1988, in Cooling Flows in Clusters and Galaxies, ed. A.C. Fabian (Dordrecht:Kluwer)

D’Ercole, A., Renzini, A., Ciotti, L., Pellegrini, S., 1989, Ap. J. (Lett),341,L9.

Ford, H.C., Butcher, H., 1979, Ap. J. Supp.,41, 147.

Heckman, T.M, Baum, S.A., van Breugel, W.J.M., McCarthy, P., 1989, Ap. J., 338, 48.

Hu, E. 1988, in Cooling Flows in Clusters and Galaxies, ed. A.C. Fabian (Dordrecht:Kluwer)

Jura, M., et al. 1987, Ap. J. (Lett), 312,L11

Kochhar, R.K., 1989, Astrophys. and Space Sci., 157, 305.

Malin, D.F., 1985, in New Aspects of Galaxy Photometry ed. J.-L. Nieto,(NY:Springer)

Matilsky, T., Jones, C., Forman, W., 1985, Ap. J.,291, 621.

Phillips, M.M., Jenkins, C.R., Dopita, M.A., Sadler, E.M., Binette, L., 1986, A.J.,81, 1062.

Schreier, E.J., Gorenstein, P., Feigelson, E.D., 1982, Ap.J., 261, 45.

Sparks, W.B., Macchetto, F., and Golombek, D. , 1989, Ap.J., 345, 153.

${ }^{2}$ Affiliated with the Astrophysics Division, Space Science Department, European Space Agency (ESA).

- Operated by the Association of Universities for Research in Astronomy. Inc., under contract with the National Aeronautics and Space Administration. 


\section{DISCUSSION}

Carilli: What about magnetic fields?

Sparks: Laing \& Bridle (1987 MNRAS 228, 557) resolved out rotation-measure structure elsewhere in the Virgo cluster, suggesting ordered fields on scales $\leqslant 2 \mathrm{kpc}$. Ordered fields don't make much difference to conduction. Rosner \& Tucker (1989 Ap. J. 338, 761) argued that even if fields are tangled, conduction can still be important. 